\title{
Cosmic magnetic fields in clusters of galaxies and their analysis
}

\author{
Joern Geisbuesch and Paul Alexander \\ Astrophysics Group, University of Cambridge, Cambridge, CB3 0HE, UK \\ email: joern@mrao.cam.ac.uk
}

\begin{abstract}
We discuss how measurements of linear polarisation of cluster background and embedded sources can be used to study cluster magnetic fields via Faraday rotation. We make forecasts for up-coming radio instruments on the basis of synthetic radio sky observations. Our mock polarised sky is modelled to agree with the sparse available data. By applying Bayesian statistical analysis methods, such as Markov Chain Monte Carlo and nested sampling techniques, we investigate which constraints can be placed on cluster magnetic field properties.
\end{abstract}

Keywords. Galaxies: clusters: general - galaxies: magnetic fields - magnetic fields - radio continuum: galaxies - polarization - methods: data analysis

\section{The polarised source sky and clusters as Faraday screens}

A powerful tool for studying magnetic fields in a variety of environments is to use Faraday rotation against background and embedded polarised sources. These source populations consist of normal and starburst galaxies as well as different types of radio AGN. To obtain realistic models of radio source populations, we use the publicly available SKADS synthetic radio source catalogue (Wilman et al. 2008). The catalogue is derived from semi-empirical simulations of different populations of radio continuum sources and extends down to faint flux limits in order to allow observation simulations of high-sensitive future radio facilities, such as the SKA and LOFAR. To determine the degree of polarisation of individual sources, we base our modelling on statistics of the NVSS catalogue (Condon et al. 1998) as derived by Tucci et al. (2003) and semi-analytic source models. Apart from semi-analytic predictions of probability distributions of fractional source polarisations, we fit the observed $1.4 \mathrm{GHz}$ distribution at the high fractional polarisation tail by a lognormal function. Moreover, we adopt the depolarisation correction recipe of Tucci et al. (2003) to obtain the intrinsic source polarisation. A map of Stokes $U$ of a simulated polarised sky realisation is shown in Figure 1a.

Observed magnetic field strengths in clusters are typically $0.1-1 \mu \mathrm{G}$. Cooling flow clusters have up to an order of magnitude stronger fields (see e.g. Taylor \& Carilli 2002). However, observational data on the structure of magnetic fields in clusters is presently rather sparse. To simulate cluster Faraday profiles we utilise a power law spectrum to model correlations of magnetic field amplitudes on different scales. The spectrum is cut on a scale below which the magnetic field strength shows no spatial fluctuations. Moreover, the cluster magnetic vector field is modelled in a divergence-free manner. For the electron density distribution we adopt a King profile and also scale the magnetic field strength in a similar fashion (i.e. decreasing with cluster radius). Figure 1b shows a Faraday depth image of a cluster for a magnetic field power spectrum of Kolmogorov type. To model the cluster number and distribution within an observed patch of sky, we employ N-body simulations. 

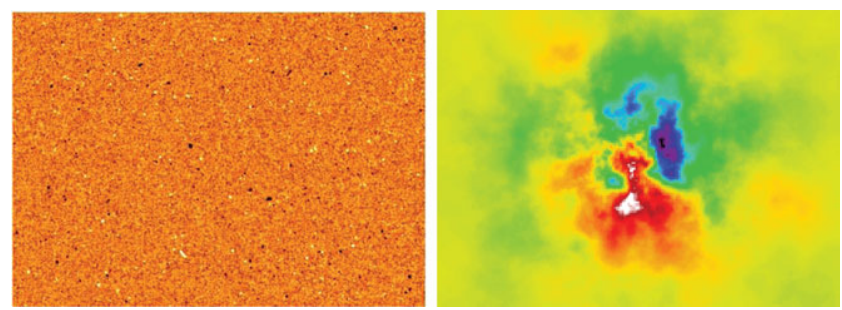

Figure 1. (a) Sky simulation of linearly polarised sources - Stokes $U$ is shown. (b) Faraday depth profile of a galaxy cluster.
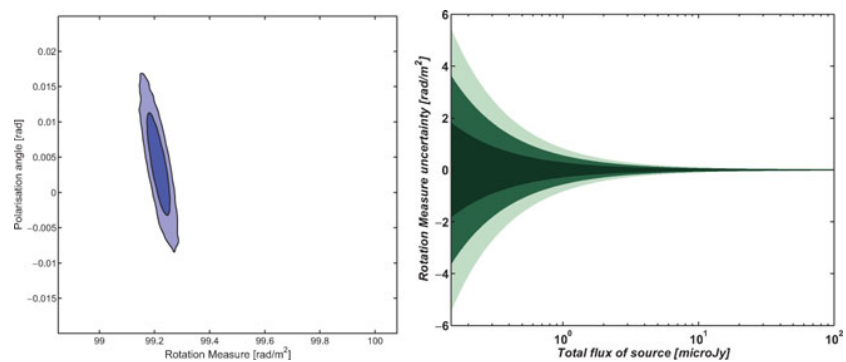

Figure 2. (a) Confidence contours for the foreground RM value and the intrinsic source polarisation angle. The dark blue shaded area gives the $68 \%$ and the light blue shaded one the $95 \%$ confidence region. (b) Uncertainty on the reconstructed RM of the foreground screen.

\section{Rotation Measure grids and Bayesian analysis}

Cluster X-ray or SZ data in combination with rotation measure (RM) observations can yield an estimate of cluster magnetic fields (see e.g. Geisbuesch et al. 2008). However, current RM grids are sparse and the sample of clusters which have a larger number of polarisation measurements of embedded or background sources is small. The SKA with its unique collection area reaches unmatched sensitivities for reasonable integration times. Thus, the number of source detections within a cluster field will significantly increase.

To investigate the potential of future SKA data for determining source intrinsic and foreground screen magneto-ionic (Faraday) properties, we model SKA mid-band observations based on the latest SKA reference designs. We apply then Bayesian techniques based on Markov Chain Monte Carlo and nested sampling methods to the simulated data to explore model posterior distributions and to evaluate model evidences (see e.g. Skilling 2006 for a detailed description of the techniques). We find that for our simulated SKA linear polarisation data the model selection ratio gives reliable information on the projected cluster line-of-sight Faraday depth even for less bright background sources with high fractional polarisation. In Figure $2 \mathrm{a}$ and $2 \mathrm{~b}$ confidence contours and reconstruction uncertainties are shown.

\section{References}

Condon, J., Cotton, W., Greisen, E., Yin, Q., Perley, R., Taylor, G., \& Broderick J. 1998, AJ 115,1693

Geisbuesch, J., Alexander, P., Krause, M., \& Bolton R. 2008, Il Nuovo Cimento, 122 B

Skilling, J. 2006, Bayesian Analysis, vol. 4, p. 833

Taylor, G. B. \& Carilli, C. L. 2002, ARA\&A 40, 319

Tucci, M., Martinez-Gonzalez, E., Toffolatti, L., Gonzalez-Nuevo, J., \& de Zotti, G. 2003, NewAR 47, 1135

Wilman, R., Miller, L., Jarvis, M., Mauch, T., Levrier, F., Abdalla, F., Rawlings, S., Kloeckner, H.-R., Obreschkow, D., Olteanu, D., \& Young, S. 2008, MNRAS 388, 1335 